\title{
Upregulation of Arp2 expression is associated with the prognosis and prediction of lymph node metastasis in bladder urothelial carcinoma
}

This article was published in the following Dove Press journal: Cancer Management and Research

\author{
Ning $\mathrm{Xu}^{*}$ \\ Gen-Yi Qu* \\ Yu-Peng Wu* \\ Xiao-Dong Li \\ Min-Yi Lin \\ Shao-Hao Chen \\ Xue-Yi Xue \\ Yong Wei
}

Department of Urology, The First Affiliated Hospital of Fujian Medical University, Fuzhou, People's Republic of China

*These authors contributed equally to this work
Correspondence: Yong Wei; Xue-Yi Xue Department of Urology, The First Affiliated Hospital of Fujian Medical University, 20 Chazhong Road, Fuzhou 350005, People's Republic of China Tel +8659187981687 Email weiyong2017@fjmu.edu.cn; xuexueyi@fjmu.edu.cn
Objective: Upregulation of actin-related protein 2/3 complex subunit 2 (Arp2) is observed in various tumors, but its expression pattern has not been revealed in bladder urothelial carcinoma (BUC). The purpose of this study was to investigate the role of Arp2 expression in the pathological features and the prognosis as well as lymph node metastasis of BUC.

Methods: A total of 228 tissue specimens from BUC patients who underwent a radical cystectomy were collected. In addition, 40 lymph node specimens and 40 normal bladder tissue specimens as controls were used. All of the specimens were used to construct a tissue microarray and were subsequently subjected to immunohistochemical staining for Arp2 expression. Logistic and Cox regression analyses and Kaplan-Meier curve analysis were applied to analyze the relation between Arp2 expression and multiple clinical features of patients with BUC.

Results: Immunohistochemical staining observation showed that Arp2 was mainly expressed in the cytoplasm and nucleus of positive cells and that Arp2 expression in BUC was significantly higher than that in normal bladder tissues. Arp2 expression in BUC tissues was associated with tumor size, tumor multiplicity, tumor stage, tumor grade, and lymph node metastasis $(P<0.05)$. Logistic regression analysis demonstrated that Arp2 expression was an independent risk factor for lymph node metastasis of BUC $(P<0.05)$. Kaplan-Meier curve analysis showed that increased Arp2 expression was associated with a shortened recurrence-free survival (RFS) and overall survival (OS) of BUC patients after radical cystectomy $(P<0.05)$.

Conclusion: These findings suggest that Arp2 is significantly upregulated in BUC tissues when compared with normal bladder tissues, and that Arp2 expression is an independent predictor for lymph node metastasis, RFS, and OS.

Keywords: Arp2, bladder urothelial carcinoma, immunohistochemistry, lymph node metastasis, prognosis

\section{Introduction}

Globally, it has been reported that nearly 43 million people are diagnosed with bladder cancer every year, contributing to $>165,000$ cancer-related deaths. ${ }^{1}$ Among bladder malignancies, $\sim 90 \%$ of cases are diagnosed as bladder urothelial carcinoma (BUC). Lymph node metastasis is the main path for BUC metastasis, and BUC metastasis to the pelvic lymph node is often observed first. Stein and Skinner demonstrated that the lymph node metastasis rate among patients who have undergone a radical cystectomy with pelvic lymph node dissection (PLND) is $13-40 \%{ }^{2}$ Therefore, prediction of lymph node metastasis before surgery could help medical oncologists to evaluate the prognosis and apply the appropriate treatment. To date, as a preoperative diagnostic method, computed tomography or magnetic resonance 
imaging remains the routine method to identify lymph node metastasis, based on the examined size and shape of the lymph nodes. However, a few cases of lymph node metastasis and lymph node inflammatory hyperplasia are often misdiagnosed or missed by magnetic resonance imaging or computed tomography.

Lymph node metastasis in BUC patients generally indicates a poor prognosis. Braud et $\mathrm{al}^{3}$ revealed the survival rate of patients who underwent radical cystectomy with PLND was examined. The long-term survival rate for patients with regional lymph node metastasis is $20 \%$ lower than that of patients without regional lymph node metastasis. ${ }^{3}$ Lymph node metastasis is a complicated process, and the underlying mechanism remains unclear. The risk factors for lymph node metastasis include tumor stage, tumor grade, and clinicopathological characteristics. ${ }^{4}$ In order to find a way to predict lymph node metastasis accurately before surgery, Karakiewicz et $\mathrm{al}^{5}$ have analyzed certain clinical data of preoperative patients and the postoperative pathological specimens of pT (3-4) and pN (1-3) stages, revealing that preoperative T stage and $\mathrm{G}$ grade, accompanied with carcinoma in situ, are independent risk factors for lymph node metastasis, whereas Green et $\mathrm{al}^{6}$ have shown that the preoperative $\mathrm{T}$ stage and the imaging examination are important factors for predicting lymph node metastasis.

Actin-related protein 2/3 complex subunit 2 (Arp2) is a member of the actin-related protein $2 / 3$ complex (ARP2/3) family, which contains the following seven members: Arp2, Arp3, ARPC1, ARPC2, ARPC3, ARPC4, and ARPC5. ${ }^{7}$ It has been demonstrated that the ARP $2 / 3$ complex functions to activate actin filaments, to mediate the assembly of actin in response to extracellular signals, as well as to monitor and regulate actin polymerization. Meanwhile, Arp2 and Arp3 provide nucleation sites for actin ${ }^{8,9}$ and also play an important role in the formation of dendritic filopodia and lamellipodia. ${ }^{10}$ The biological behaviors of tumors, such as cell proliferation, migration, and invasion, are closely linked to the actin skeleton and filopodia formation. ${ }^{11}$ In the preliminary study, the increased expression level of Arp2 in 10 pairs of BUC specimens compared to normal bladder tissues (data not shown) has also been confirmed. Therefore, this study aimed to investigate Arp2 expression in BUC and to compare the expression patterns between normal bladder tissues and lymph node specimens. Then, the association of Arp2 expression with the clinicopathological characteristics and lymph node metastasis in BUC patients was revealed.

\section{Methods}

\section{Tissue specimens and data collection}

Tissue specimens from 288 patients with BUC who had undergone a radical cystectomy with PLND were collected between January 2007 and January 2011. The anatomical limits of the lymphadenectomy were the genitofemoral nerve (laterally), the obturator nerve (medially), and the node of Cloquet (distally) according to the previous description. ${ }^{20}$ Specimens were fixed with $4 \%$ formalin, embedded in paraffin blocks, and confirmed by a pathological examination. Patients were diagnosed histologically and did not receive any adjuvant chemotherapy or radiotherapy preoperatively. Moreover, 40 pairs of normal bladder urethral tissue and lymph node of the BUC specimens were obtained. The normal bladder tissues were those pathologically confirmed normal bladder mucosa, which were retrieved $5 \mathrm{~cm}$ away from the bladder cancer region via radical cystectomy. ${ }^{21,22} \mathrm{Clinical}$ data include age, gender, tumor stage, tumor grade, tumor size, number of tumors, and pelvic lymph nodes.

\section{Construction of tissue microarray}

Paraffin blocks from BUC and normal bladder tissues were retrieved and sectioned for hematoxylin and eosin staining to confirm the diagnosis and to identify the representative areas for TMA construction. By using a self-designed TMA maker, cores of $2 \mathrm{~mm} \times 2 \mathrm{~mm}$ size were generated in a new paraffin block, and for each paraffin block, $5 \times 8$ TMAs were prepared. Afterward, the paraffin block was then sectioned into $4 \mu \mathrm{m}$-thick tissue sections for immunohistochemical staining of Arp2 protein.

\section{Immunohistochemistry}

The sections were conventionally dewaxed and rehydrated. Antigen retrieval was performed using high-pressure heating in $0.1 \mathrm{M}$ citric acid buffer ( $\mathrm{pH}$ 5.0; Fuzhou Maixin Biotech. Co., Ltd., Fuzhou, People's Republic of China). After that, to block endogenous peroxidase activity, the TMAs were then incubated with $3 \% \mathrm{H}_{2} \mathrm{O}_{2}$ for 10 min followed by $20 \%$ normal goat serum at room temperature for $30 \mathrm{~min}$. Microarray sections were incubated with a rabbit monoclonal anti-Arp2 antibody (Cell Signaling Technology, Danvers, MA, USA) at a dilution of $1: 400$ at $4{ }^{\circ} \mathrm{C}$ overnight. On the next day, the sections were washed briefly with phosphate-buffered saline (PBS) three times and further incubated with a secondary antibody (Dako Denmark A/S, Glostrup, Denmark) at $37^{\circ} \mathrm{C}$ for $30 \mathrm{~min}$ and subsequently examined with a ChemMate EnVision Detection Kit (Dako Denmark A/S). The sections 
were subjected to a colorimetric reaction with 3,3'-diaminobenzidine solution, counterstained briefly with hematoxylin, and mounted with a cover slip.

\section{Determination of Arp2 expression}

Positively confirmed sections were used as a positive control, and sections treated with PBS were used as a negative control. The immunostained TMA sections were separately inspected and scored by two pathologists under a light microscope (Olympus Corporation, Tokyo, Japan). Brown granular substance (Arp2) was observed in the cytoplasm and nucleus of positive cells. A semiquantitative method was conducted, and the total Arp2 immunostaining score was set according to the staining intensity and the proportion of positive cells. The staining intensity $(I)$ was recorded as 0 (absent), 1 (weak; light yellow or only individual cells were stained yellow to brown), 2 (moderate; staining intensity between 1 point and 3 points), or 3 (strong; yellow to brown staining), whereas the proportion $(P)$ of positive cells was recorded as $0(<5 \%)$, $1(5-25 \%), 2(26-50 \%), 3(51-75 \%)$, or $4(>75 \%)$. The final Arp2 expression score was calculated using the value of the proportion of positive cell score multiplied by the staining intensity score as “-” (score, 0-1), “+” (score, 2-3), “++" (score, 4-5), or " +++ " (score, $\geq 6)$. $^{23}$

\section{Statistical methods}

SPSS 22.0 statistical software (IBM Corporation, Armonk, NY, USA) was utilized to perform all statistical analyses. The Mann-Whitney $U$ rank-sum test was applied for comparisons of Arp2 expression in BUC tissue and normal bladder tissue specimens (Table 1). The Mann-Whitney $U$ rank-sum test was used for comparisons of clinicopathological features of BUC patients associated with Arp2 expression (Table 2). The Chi-squared test or Fisher's exact test was applied for comparisons of clinicopathological features of BUC associated with lymph node metastasis (Table 3). The Mann-Whitney $U$ rank-sum test was used

Table I Arp2 expression in BUC specimens vs paired normal bladder tissue, and positive lymph node specimens

\begin{tabular}{llllllll}
\hline Group & \multicolumn{3}{l}{ Arp2 expression } & & Positive & P value \\
\cline { 2 - 5 } & - & + & ++ & +++ & rate (\%) & \\
\hline Normal bladder tissue & 31 & 6 & 3 & 0 & 22.5 & $<0.001$ \\
BUC & 9 & 12 & 12 & 7 & 77.5 & \\
Positive lymph nodes & 5 & 4 & 16 & 15 & 87.5 & \\
\hline
\end{tabular}

Notes: BUC vs normal bladder tissue, $P<0.01$; positive lymph nodes vs BUC, $P<0.05$; positive lymph nodes vs normal bladder tissue, $P<0.01$.

Abbreviations: Arp2, actin-related protein 2/3 complex subunit 2; BUC, bladder urothelial carcinoma. for the comparisons of Arp2 expression and lymph node metastasis (Table 3). Lymph node metastasis predictors were analyzed using multivariate logistic regression analysis (Table 4). Univariate and multivariate Cox proportional hazard models were used to evaluate the predictive value of each indicator for the BUC 5-year recurrence-free survival (RFS) (Table 5) and overall survival (OS) (Table 6). Kaplan-Meier plots and the log-rank test were performed to assess the association of Arp2 expression with the 5 -year RFS and the OS. $P<0.05$ was considered statistically significant.

\section{Ethics}

This study was approved by the ethics committee of The First Affiliated Hospital of Fujian Medical University (Fuzhou, Fujian, People's Republic of China) (approval no: 2015123). Written informed patient consent for the use of the tissue specimen and participation in the study was obtained from each recruited patient.

\section{Results}

\section{Arp2 is differentially expressed in BUC and normal bladder tissue specimens}

Initially, the clinical information, including age, gender, tumor size, number of tumors, tumor grade, tumor stage, and pelvic lymph nodes, for all the clinical samples collected are summarized in Table 7. Since Arp2 protein was mainly expressed in the cytoplasm of positive cells, to establish a link between the clinical data and Arp2 expression, Arp2 expression was examined by immunohistochemical staining of normal bladder tissues and BUC tissues, respectively. The results showed that Arp2 expression was significantly higher in BUC tissues than in normal bladder tissues (77.5 vs $22.50 \%$; $P<0.05$; Table 1, Figure 1).

\section{Arp2 expression is associated with clinicopathological data in BUC patients}

Next, the expression of Arp2 protein was matched with different clinicopathological data of the BUC patients (summarized in Table 7). It was found that a higher Arp2 expression was associated with tumor size, number of tumors, tumor grade, tumor stage, and lymph node metastasis $(P<0.05$, Table 2$)$, whereas there was little association between Arp2 expression and age or gender of the patients $(P>0.05$, Table 2$)$. Further observation of immunohistochemical staining indicated that Arp2 expression was more commonly observed in largesize tumors, multiple tumors, high-grade tumors, late-stage 
Table 2 Association of Arp2 expression with clinicopathological features of bladder urothelial carcinoma patients

\begin{tabular}{|c|c|c|c|c|c|c|}
\hline \multirow[t]{2}{*}{ Variable } & \multirow[t]{2}{*}{$\mathbf{N}$} & \multicolumn{4}{|c|}{ Arp2 expression } & \multirow[t]{2}{*}{$P$ value } \\
\hline & & - & + & ++ & +++ & \\
\hline Total, n (\%) & 228 & $39(17.1)$ & $47(20.6)$ & $81(35.5)$ & $61(26.8)$ & \\
\hline \multicolumn{7}{|l|}{ Age, years } \\
\hline$<60$ & 110 & $23(20.9)$ & $20(18.2)$ & $36(32.7)$ & $31(28.2)$ & 0.391 \\
\hline$\geq 60$ & 118 & $16(13.6)$ & $27(22.9)$ & $45(38.1)$ & $30(25.4)$ & \\
\hline \multicolumn{7}{|l|}{ Gender } \\
\hline Male & 182 & $31(17.0)$ & 35 (19.2) & $66(36.3)$ & $50(27.5)$ & 0.768 \\
\hline Female & 46 & $8(17.4)$ & $12(26.1)$ & $15(32.6)$ & II (23.9) & \\
\hline \multicolumn{7}{|c|}{ Tumor size, cm } \\
\hline$<3$ & 104 & $32(30.8)$ & $26(25.0)$ & $29(27.9)$ & $17(16.3)$ & $<0.001$ \\
\hline$\geq 3$ & 124 & $7(5.7)$ & $21(16.9)$ & $52(41.9)$ & $44(35.5)$ & \\
\hline \multicolumn{7}{|l|}{ Multiplicity } \\
\hline Single & 89 & $21(23.6)$ & $10(11.2)$ & $27(30.3)$ & 31 (34.8) & 0.002 \\
\hline Multiple & 139 & $18(13.0)$ & $37(26.6)$ & $54(38.8)$ & $30(21.6)$ & \\
\hline \multicolumn{7}{|c|}{ Tumor grade, n (\%) } \\
\hline Low & 78 & $20(25.7)$ & $24(30.8)$ & $20(25.6)$ & $14(17.9)$ & $<0.001$ \\
\hline High & 150 & $19(12.7)$ & $23(15.3)$ & $6 \mathrm{I}(40.7)$ & $47(31.3)$ & \\
\hline \multicolumn{7}{|c|}{ Clinical stage (cT), n (\%) } \\
\hline $\mathrm{TI}-\mathrm{T} 2$ & 106 & $31(29.2)$ & $26(24.5)$ & $29(27.4)$ & $20(18.9)$ & $<0.001$ \\
\hline T3-T4 & 122 & $8(6.6)$ & $21(17.2)$ & $52(42.6)$ & $41(33.6)$ & \\
\hline \multicolumn{7}{|c|}{ Lymph node status } \\
\hline Negative & 157 & $33(21.0)$ & $4 I(26.1)$ & $50(31.9)$ & $33(21.0)$ & $<0.001$ \\
\hline Positive & 71 & $6(8.5)$ & $6(8.5)$ & $31(43.7)$ & $28(39.3)$ & \\
\hline
\end{tabular}

Abbreviation: Arp2, actin-related protein 2/3 complex subunit 2.

Table 3 Association of clinicopathological features with lymph node metastasis of bladder urothelial carcinoma

\begin{tabular}{|c|c|c|c|c|}
\hline \multirow[t]{2}{*}{ Variables } & \multirow[t]{2}{*}{$\mathbf{N}$} & \multicolumn{2}{|c|}{ Lymph node metastasis } & \multirow[t]{2}{*}{$P$ value } \\
\hline & & Negative & Positive & \\
\hline Total, n (\%) & 228 & $157(68.9)$ & 7I (3I.I) & \\
\hline \multicolumn{5}{|l|}{ Age, years } \\
\hline$<60$ & 110 & $80(72.7)$ & $30(27.3)$ & 0.253 \\
\hline$\geq 60$ & 118 & $77(65.3)$ & $41(34.7)$ & \\
\hline \multicolumn{5}{|l|}{ Gender } \\
\hline Male & 182 & $123(67.6)$ & $59(32.4)$ & 0.478 \\
\hline Female & 46 & $34(73.9)$ & $12(26.1)$ & \\
\hline \multicolumn{5}{|c|}{ Tumor size, $\mathrm{cm}$} \\
\hline$<3$ & 104 & $85(81.7)$ & $19(18.3)$ & $<0.001$ \\
\hline$\geq 3$ & 124 & $72(58.1)$ & $52(41.9)$ & \\
\hline \multicolumn{5}{|l|}{ Multiplicity } \\
\hline Single & 89 & $69(77.5)$ & $20(22.5)$ & 0.028 \\
\hline Multiple & 139 & $88(63.3)$ & $51(36.7)$ & \\
\hline \multicolumn{5}{|c|}{ Tumor grade, n (\%) } \\
\hline Low & 78 & $65(83.3)$ & $13(16.7)$ & 0.001 \\
\hline High & 150 & $92(61.3)$ & $58(38.7)$ & \\
\hline \multicolumn{5}{|c|}{ Clinical stage (cT), n (\%) } \\
\hline $\mathrm{TI}-\mathrm{T} 2$ & 106 & $88(83.0)$ & $18(17.0)$ & $<0.001$ \\
\hline T3-T4 & 122 & $69(56.6)$ & $53(43.4)$ & \\
\hline \multicolumn{5}{|c|}{ Arp2 expression } \\
\hline- & 39 & $33(84.6)$ & $6(15.4)$ & $<0.001$ \\
\hline+ & 47 & $4 I(87.2)$ & $6(12.8)$ & \\
\hline++ & 81 & $50(6 \mid .7)$ & $31(38.3)$ & \\
\hline +r+ & 61 & $33(54.1)$ & $28(45.9)$ & \\
\hline
\end{tabular}

Abbreviation: Arp2, actin-related protein 2/3 complex subunit 2 .
Table 4 Multivariate analysis of clinicopathological features for association with lymph node metastasis of BUC

\begin{tabular}{lll}
\hline Variable & OR (95\% Cl) & P value \\
\hline Tumor size (<3 vs $\geq 3)$ & $1.550(0.746-3.22 \mathrm{I})$ & $0.24 \mathrm{I}$ \\
Multiplicity (single vs multiple) & $\mathrm{I} .858(0.935-3.694)$ & 0.077 \\
Tumor grade (low vs high) & $\mathrm{I} .44 \mathrm{I}(0.649-3.200)$ & 0.370 \\
Clinical stage (cT) (TI-T2 vs T3-T4) & $\mathrm{I} .986(0.972-4.059)$ & $0.02 \mathrm{I}$ \\
Arp2 (-l+/++/+++) & $\mathrm{I} .66 \mathrm{I}(\mathrm{I} .612-2.373)$ & 0.005 \\
\hline
\end{tabular}

Abbreviations: Arp2, actin-related protein 2/3 complex subunit 2; BUC, bladder urothelial carcinoma.

tumors, and lymph node-positive BUC tissue specimens (Figure 2).

\section{Arp2 expression and clinicopathological factors are associated with pelvic lymph node metastasis of BUC}

Next, subgroup analyses were performed to investigate the association between Arp2 expression and clinicopathological factors as well as pelvic lymph node metastasis of BUC. The results showed that BUC metastasis to the pelvic lymph nodes was associated with Arp2 expression, tumor size, number of tumors, tumor grade, and tumor stage $(P<0.05$; Table 3$)$ but not related to the age or gender $(P>0.05$; Table 3$)$ of the patients. Multivariate logistic regression analysis further 
Table 5 Univariate and multivariate Cox proportional hazard analyses for the 5-year recurrence-free survival

\begin{tabular}{|c|c|c|c|c|}
\hline \multirow[t]{2}{*}{ Variables } & \multicolumn{2}{|l|}{ Univariate analysis } & \multicolumn{2}{|c|}{ Multivariate analysis } \\
\hline & HR (95\% Cl) & $P$ value & HR (95\% Cl) & $P$ value \\
\hline \multicolumn{5}{|l|}{ Univariate analysis } \\
\hline Age (years, $<60$ vs $\geq 60$ ) & $1.222(0.789-1.892)$ & 0.369 & & \\
\hline Gender (male vs $>25$ ) & $1.306(0.788-2.164)$ & 0.301 & & \\
\hline Tumor size ( $<3$ vs $\geq 3$ ) & $1.724(1.908-2.707)$ & 0.018 & $1.072(0.656-1.750)$ & 0.782 \\
\hline Multiplicity (single vs multiple) & $1.314(0.834-2.069)$ & 0.240 & & \\
\hline Tumor grade (low vs high) & $1.324(0.826-2.122)$ & 0.244 & & \\
\hline Clinical stage (cT) (TI-T2 vs T3-T4) & $2.018(1.280-3.181)$ & 0.002 & $\mathrm{I} .398(0.85 \mathrm{I}-2.295)$ & 0.186 \\
\hline Lymph node status (negative vs positive) & $2.333(1.434-3.476)$ & $<0.001$ & $1.767(1.120-2.789)$ & 0.014 \\
\hline $\operatorname{Arp} 2(-/+/++/+++)$ & $1.710(1.348-2.171)$ & $<0.001$ & $1.593(1.234-2.507)$ & $<0.001$ \\
\hline
\end{tabular}

Abbreviation: Arp2, actin-related protein 2/3 complex subunit 2.

Table 6 Univariate and multivariate Cox proportional hazard analyses for the 5-year overall survival

\begin{tabular}{|c|c|c|c|c|}
\hline \multirow[t]{2}{*}{ Variables } & \multicolumn{2}{|l|}{ Univariate analysis } & \multicolumn{2}{|c|}{ Multivariate analysis } \\
\hline & HR (95\% CI) & $P$ value & HR (95\% Cl) & $P$ value \\
\hline \multicolumn{5}{|l|}{ Univariate analysis } \\
\hline Age (years; $<60$ vs $\geq 60$ ) & $1.370(0.855-2.194)$ & 0.191 & & \\
\hline Gender (male vs $>25$ ) & $1.436(0.849-2.430)$ & 0.177 & & \\
\hline Tumor size $(<3$ vs $\geq 3)$ & $1.954(1.195-3.196)$ & 0.008 & $\mathrm{I} .068(0.63 \mathrm{I}-\mathrm{I} .808)$ & 0.807 \\
\hline Multiplicity (single vs multiple) & $1.484(0.902-2.440)$ & 0.120 & & \\
\hline Tumor grade (low vs high) & $1.608(0.950-2.720)$ & 0.077 & & \\
\hline Clinical stage (cT) (TI-T2 vs T3-T4) & $2.628(1.577-4.377)$ & $<0.001$ & $1.745(1.011-3.012)$ & 0.046 \\
\hline Lymph node status (negative vs positive) & $2.945(1.845-4.699)$ & $<0.001$ & $2.268(1.406-3.659)$ & 0.001 \\
\hline $\operatorname{Arp} 2(-1+/++/+++)$ & $1.937(1.482-2.531)$ & $<0.001$ & $1.796(1.344-2.398)$ & $<0.001$ \\
\hline
\end{tabular}

Abbreviation: Arp2, actin-related protein 2/3 complex subunit 2.

Table 7 Clinicopathological characteristics of 228 patients with bladder urothelial carcinoma

\begin{tabular}{ll}
\hline Clinicopathological characteristics & Value \\
\hline Age, years & \\
Mean \pm SD & $57.52 \pm 9.10$ \\
Range & $33-77$ \\
Gender & \\
Male & $182(79.8)$ \\
Female & $46(20.2)$ \\
Tumor size, cm & \\
Mean \pm SD & $3.29 \pm 1.41$ \\
Range & $0.6-6.3$ \\
Multiplicity & \\
Single & $89(39.0)$ \\
Multiple & $139(61.0)$ \\
Tumor grade, $\mathrm{n}(\%)$ & \\
Low & $78(34.2)$ \\
High & $150(65.8)$ \\
Clinical stage (cT), $\mathrm{n}(\%)$ & \\
TI-T2 & $106(46.5)$ \\
T3-T4 & $122(53.5)$ \\
Lymph node status & \\
Negative & $157(68.9)$ \\
Positive & $71(31.1)$ \\
\hline
\end{tabular}

revealed that Arp2 expression and clinical tumor $\mathrm{T}$ stage were independent risk factors for BUC lymph node metastasis $(P<0.05$; Table 4).

\section{Higher Arp2 expression is associated with a shorter 5-year RFS in BUC patients}

To reveal a link between the 5-year RFS and Arp2 expression, univariate Cox regression analysis was performed. Five-year RFS was calculated from the date of curative surgery to date of recurrence in 5 years. The 5-year RFS was $55.6 \%$ in this study. Local recurrence refers to the recurrence from soft tissue within the scope of radical cystectomy, and the distant metastasis refers to the recurrence beyond the pelvic cavity. The results indicated that the 5-year RFS of the BUC patients was significantly associated with an increased Arp2 expression and multiple clinical features, including tumor size, clinical $\mathrm{T}$ stage, and lymph node metastasis $(P<0.05$; Table 5). The data clearly indicated that the higher the Arp2 expression, the 

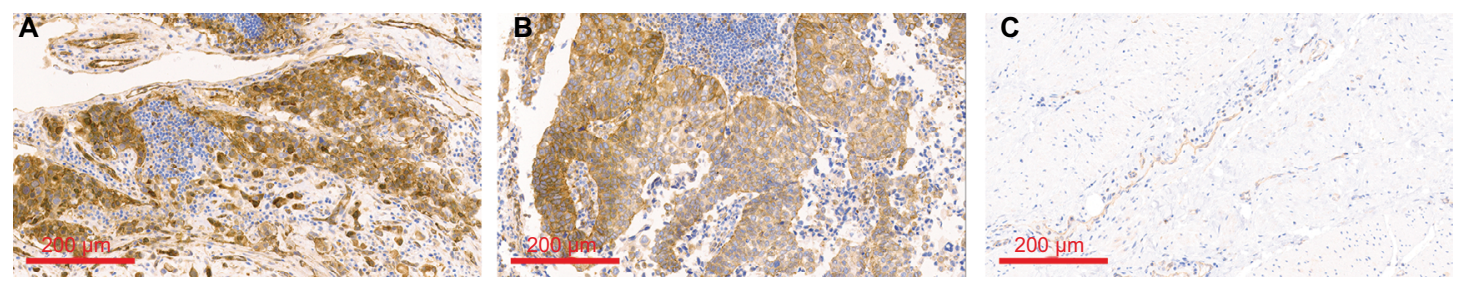

Figure I Tissue microarrays containing BUC and normal bladder tissues were immunostained with a monoclonal anti-Arp2 antibody, and the data were semiquantitatively analyzed: (A) positive lymph nodes; (B) BUC; and (C) normal bladder tissue; 200× magnification.

Note: The expression of Arp2 in the positive lymph nodes was higher than that in the paired BUC, and the expression of Arp2 in the BUC was higher than that in the paired normal bladder tissue.

Abbreviations: Arp2, actin-related protein 2/3 complex subunit 2; BUC, bladder urothelial carcinoma.
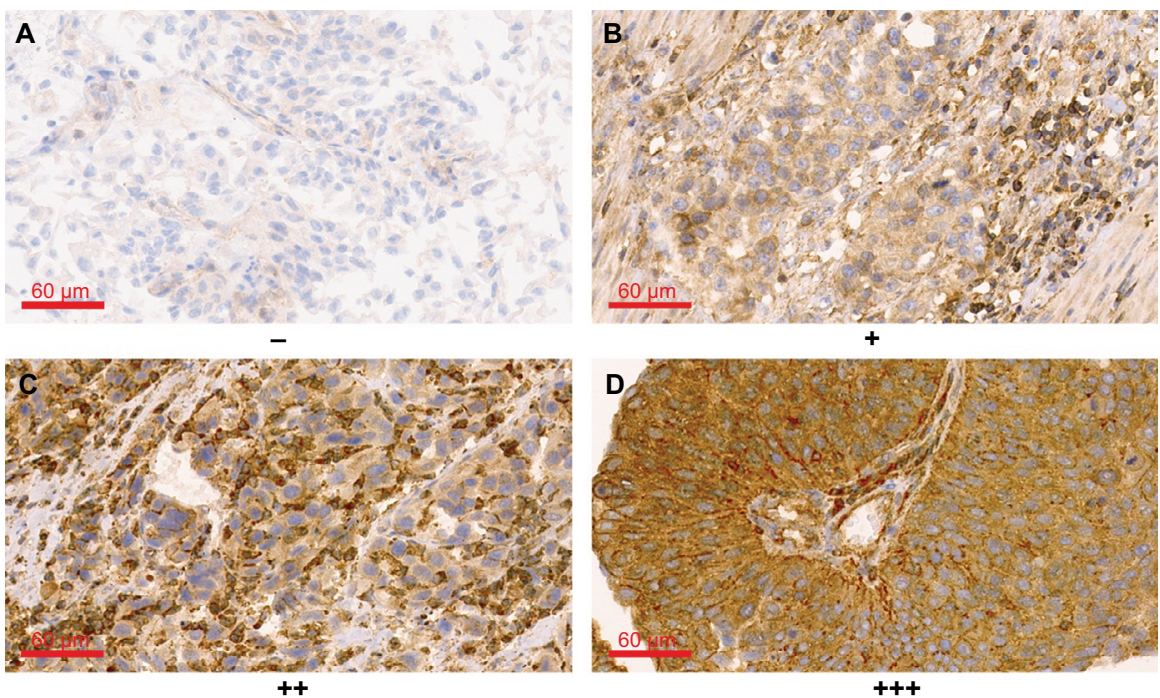

Figure 2 Different expression levels of Arp2 protein in BUC (400× magnification)

Notes: (A) Negative (score of 0), (B) weakly positive (score of 2), (C) positive (score of 5), and (D) strongly positive (score of 7 ) Arp2 protein expression.

Abbreviations: Arp2, actin-related protein 2/3 complex subunit 2; BUC, bladder urothelial carcinoma.

shorter the 5-year RFS ( $P<0.05$; Table 5); in addition, the same conclusion was drawn from the Kaplan-Meier curve analysis (Figure $3 ; P<0.001$ ).

\section{Higher Arp2 expression is associated with a shorter 5-year OS in BUC patients}

Finally, the relationship between the 5-year OS and Arp2 expression was investigated. Five-year OS was calculated from the date of surgery to date of death from any cause in 5 years. The 5 -year OS was $67.9 \%$ in this study. Univariate Cox regression analysis indicated that some clinical features, including tumor size, clinical $\mathrm{T}$ stage, lymph node metastasis, and increased Arp2 expression, were significantly associated with the 5-year OS in the BUC patients $(P<0.05$; Table 6); in addition, a higher clinical T stage, lymph node metastasis, and increased Arp2 expression were associated with a shortened 5-year OS $(P<0.05$; Table 6). The same conclusion was drawn from Kaplan-Meier curve analysis, ie, an increased
Arp2 expression was associated with a shortened 5-year OS (Figure 4; $P<0.001$ ).

\section{Discussion}

Arp2 is a member of the Arp2/3 complex family and regulates cell migration by impacting polymerization and depolymerization of actin. Therefore, the complex is viewed as a major player in the invasion and metastasis processes of tumor cells. ${ }^{12,13}$ To date, previous studies have suggested that an increased Arp2 expression is observed in various tumors, including gastric cancer, breast tumors, colorectal tumors, lung cancer, and others. ${ }^{14-17}$ Moreover, Arp2 expression is significantly associated with the occurrence, development, invasion, metastasis, and prognosis of these cancers. ${ }^{14-17}$ These studies suggest that Arp2 expression is closely associated with tumor invasion, metastasis, and prognosis. However, few studies have been carried out to reveal the link between Arp2 expression and BUC. 


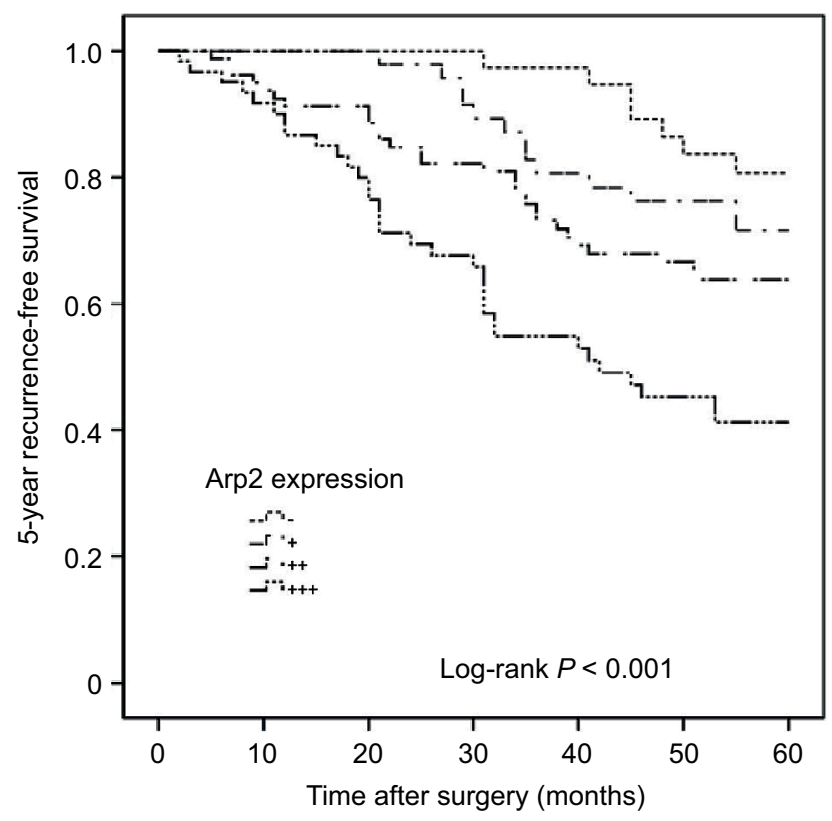

Figure 3 Kaplan-Meier curve analysis of the 5-year RFS of BUC patients stratified by Arp2 expression.

Note: Increased Arp2 expression was associated with a shortened 5-year RFS (logrank test, $P<0.001$ ).

Abbreviations: Arp2, actin-related protein 2/3 complex subunit 2; BUC, bladder urothelial carcinoma; RFS, recurrence-free survival.

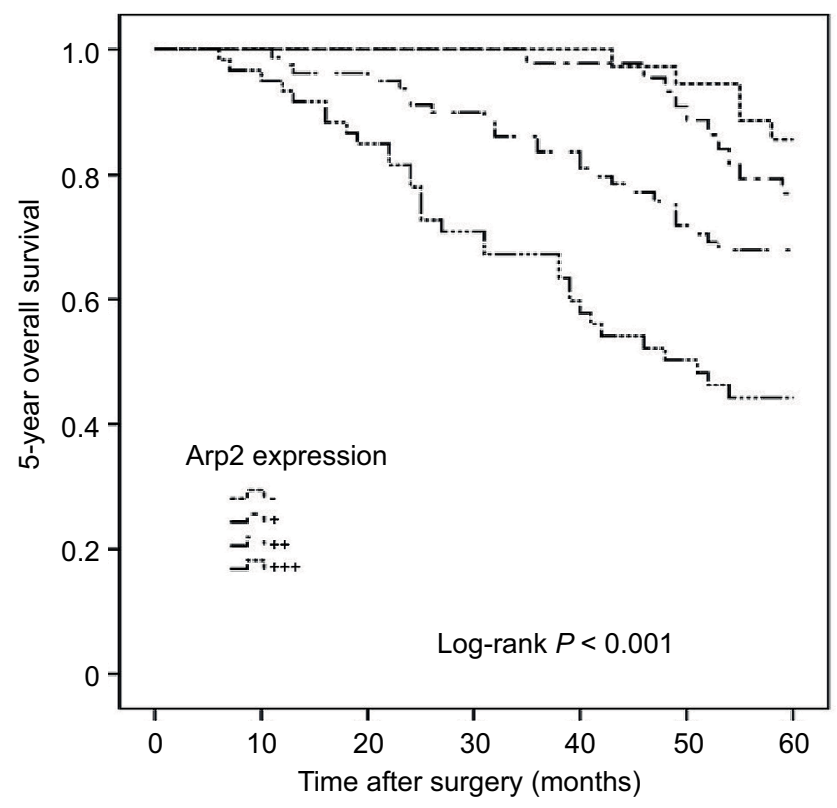

Figure 4 Kaplan-Meier curves of the 5-year OS of BUC patients stratified by Arp2 expression.

Note: Increased Arp2 expression was associated with a shortened 5-year OS (logrank test, $P<0.001$ ).

Abbreviations: Arp2, actin-related protein 2/3 complex subunit 2; BUC, bladder urothelial carcinoma; OS, overall survival.

For the first time, our study evaluated Arp2 expression in a large population of patients with bladder cancer, including 228 BUC tissues and 40 normal bladder tissues. Forty lymph node specimens were used to construct the TMA, which was subsequently subjected to immunohistochemical staining. The results showed that $82.9 \%$ of the BUC specimens had positive Arp2 expression, including 20.6\% with weak $(+)$ expression, $35.5 \%$ with moderate $(++)$ expression, and $26.8 \%$ with strong $(+++)$ expression, whereas only $22.5 \%$ of the normal bladder tissues had positive Arp2 expression. These data clearly indicate that Arp2 expression could be a potential marker for patients with BUC.

Multiple tumors that are frequently recurrent are typical features of bladder cancers. The clinical tumor stage, pathological grade, and lymph node metastasis have been shown to be important indicators for predicting the prognosis. ${ }^{18,19}$ Although numerous studies have shown that the pathological grade and clinical tumor stage play an important role in predicting the prognosis of patients with bladder cancer, in clinical practice, to determine lymph node metastasis. In this study, a significantly increased expression of Arp2 was observed in patients with a tumor of $>3 \mathrm{~cm}$ in diameter, multiple tumors, high-stage tumors, high-grade tumors, and lymph node metastasis. Moreover, it was found that Arp2 expression was related to lymph node metastasis and that an increased Arp2 expression could be an independent factor for lymph node metastasis. Since it has been revealed that the risk of lymph node metastasis in patients with an increased expression of Arp2 is 1.66-fold higher than that of patients without an increased expression of Arp2, it is clinically reasonable to conclude that Arp2 could function as a potential indicator for predicting lymph node metastasis.

Our study further explored the factors associated with the 5-year RFS and OS after radical cystectomy and standard PLND with two statistical analysis methods, which showed that the tumor size, clinical $\mathrm{T}$ stage, lymph node metastasis, and increased Arp2 expression were associated with the 5-year RFS and OS. Further analysis revealed that an increased Arp2 expression was closely associated with a shortened 5-year RFS and OS. This conclusion implicated that the expression of Arp2 is also a potential indicator for the 5-year RFS and OS for patients with BUC.

Several potential mechanisms could be involved to explain the association between expression of Arp2 and BUC carcinogenesis. Arp2 has been shown to be involved in polymerization of actin filaments after exposure to various stimuli. Cells that express Arp2 may have greater ability to move freely than cells that do not. ${ }^{17}$ In our study, the expression of Arp2 was detected at a high level in BUC cells, which indicates that the BUC cells have greater ability to move 
freely than normal bladder urothelial tissue cells. The migration ability was partly activated by the Arp 2 expression in BUC cell carcinogenesis. Tumor cells were more frequently positive for Arp2 as the invasion increased. The expression of Arp2 was also correlated with the transformation of adenomas with severe atypia into carcinomas. However, the specific mechanism of Arp2 in BUC still needs further investigation.

\section{Conclusion}

The significance of this study is that Arp2 expression could be a potential independent risk factor to predict pelvic lymph node metastasis as well as an indicator to predict the 5-year RFS and OS after a radical cystectomy with PLND.

\section{Disclosure}

The authors report no conflicts of interest in this work.

\section{References}

1. Ferlay J, Soerjomataram I, Dikshit R, et al. Cancer incidence and mortality worldwide: sources, methods and major patterns in GLOBOCAN 2012. Int J Cancer. 2015;136(5):E359-E386.

2. Stein JP, Skinner DG. Radical cystectomy for invasive bladder cancer: long-term results of a standard procedure. World $J$ Urol. 2006;24(3):296-304.

3. Braud G, Battisti S, Karam G, Bouchot O, Rigaud J. Prognostic value of lymph node dissections in bladder cancer treated with radical cystectomy. Prog Urol. 2008;18(6):351-357.

4. Jensen JB, Ulhoi BP, Jensen KM. Lymph node mapping in patients with bladder cancer undergoing radical cystectomy and lymph node dissection to the level of the inferior mesenteric artery. BJU Int. 2010;106(2):199-205.

5. Karakiewicz PI, Shariat SF, Palapattu GS, et al. Precystectomy nomogram for prediction of advanced bladder cancer stage. Eur Urol. 2006;50(6):1254-1260.

6. Green DA, Rink M, Hansen J, et al. Accurate preoperative prediction of non-organ-confined bladder urothelial carcinoma at cystectomy. $B J U$ Int. 2013;111(3):404-411.

7. Robinson RC, Turbedsky K, Kaiser DA, et al. Crystal structure of Arp2/3 complex. Science. 2001;294(5547):1679-1684.

8. Nolen BJ, Pollard TD. Structure and biochemical properties of fission yeast Arp2/3 complex lacking the Arp2 subunit. J Biol Chem. 2008;283(39):26490-26498.
9. Campellone KG, Welch MD. A nucleator arms race: cellular control of actin assembly. Nat Rev Mol Cell Biol. 2010;11(4):237-251.

10. Maritzen T, Zech T, Schmidt MR, Krause E, Machesky LM, Haucke V. Gadkin negatively regulates cell spreading and motility via sequestration of the actin-nucleating ARP2/3 complex. Proc Natl Acad Sci U S A. 2012;109(26):10382-10387.

11. LeClaire LL 3rd, Baumgartner M, Iwasa JH, Mullins RD, Barber DL. Phosphorylation of the Arp2/3 complex is necessary to nucleate actin filaments. J Cell Biol. 2008;182(4):647-654.

12. Arismendi-Morillo G, Hoa NT, Ge L, Jadus MR. Mitochondrial network in glioma's invadopodia displays an activated state both in situ and in vitro: potential functional implications. Ultrastruct Pathol. 2012;36(6):409-414.

13. Bisi S, Disanza A, Malinverno C, Frittoli E, Palamidessi A, Scita G. Membrane and actin dynamics interplay at lamellipodia leading edge. Curr Opin Cell Biol. 2013;25(5):565-573.

14. Zheng HC, Zheng YS, Li XH, et al. Arp2/3 overexpression contributed to pathogenesis, growth and invasion of gastric carcinoma. Anticancer Res. 2008;28(4b):2225-2232.

15. Iwaya K, Norio K, Mukai K. Coexpression of Arp2 and WAVE2 predicts poor outcome in invasive breast carcinoma. Mod Pathol. 2007;20(3):339-343.

16. Semba S, Iwaya K, Matsubayashi J, et al. Coexpression of actinrelated protein 2 and Wiskott-Aldrich syndrome family verprolinehomologous protein 2 in adenocarcinoma of the lung. Clin Cancer Res. 2006;12(8):2449-2454.

17. Otsubo T, Iwaya K, Mukai $Y$, et al. Involvement of Arp $2 / 3$ complex in the process of colorectal carcinogenesis. Mod Pathol. 2004;17(4):461-467.

18. Lughezzani G, Sun M, Jeldres C, et al. Adenocarcinoma versus urothelial carcinoma of the urinary bladder: comparison between pathologic stage at radical cystectomy and cancer-specific mortality. Urology. 2010;75(2):376-381.

19. Schultz L, Albadine R, Hicks J, et al. Expression status and prognostic significance of mammalian target of rapamycin pathway members in urothelial carcinoma of urinary bladder after cystectomy. Cancer. 2010;116(23):5517-5526.

20. Shao P, Meng X, Li J, et al. Laparoscopic extended pelvic lymph node dissection during radical cystectomy: technique and clinical outcomes. BJU Int. 2011;108(1):124-128.

21. Zhang B, Wang M, Gong A, et al. HucMSC-exosome mediatedWnt4 signaling is required for cutaneous wound healing. Stem Cells. 2015;33(7):2158-2168.

22. Wang HT, Ma FL, Ma XB, Han RF, Zhang YB, Chang JW. Differential gene expression profiling in aggressive bladder transitional cell carcinoma compared to the adjacent microscopically normal urothelium by microdissection-SMART cDNA PCR-SSH. Cancer Biol Ther. 2006;5(1):104-110.

23. Ma HQ, Liang XT, Zhao JJ, et al. Decreased expression of Neurensin-2 correlates with poor prognosis in hepatocellular carcinoma. World $J$ Gastroenterol. 2009; 15(38):4844-4848. 
Cancer Management and Research

\section{Publish your work in this journal}

Cancer Management and Research is an international, peer-reviewed open access journal focusing on cancer research and the optimal use of preventative and integrated treatment interventions to achieve improved outcomes, enhanced survival and quality of life for the cancer patient

The manuscript management system is completely online and includes

Submit your manuscript here: https://www.dovepress.com/cancer-management-and-research-journal 